\title{
Anime y consumo en Argentina en las páginas de Clarín, La Nación y Página 12 (1997-2001)
}

\section{Meo, Analia Lorena}

Resumen:

Este artículo aborda una primera aproximación acerca de las representaciones efectuadas por los periódicos argentinos Clarín, La Nación y Página 12 referente a la animación japonesa (アニメanime) y su consumo en Argentina durante el período 1997-2001.

Palabras clave: anime - Argentina periódicos argentinos -

representaciones - consumo.

$\left(^{*}\right)$ Doctoranda en Ciencias Sociales. Facultad de Ciencias Sociales, Universidad de Buenos Aires (FCSUBA). Becaria doctoral en el marco del proyecto de investigación UBACyT "EI Otro en los medios de comunicación. Diferencia(s) y legitimación simbólica de la(s) desigualdades" dirigido por la

\section{Cuadernos del Centro de Estudios de} Diseño y Comunicación №74

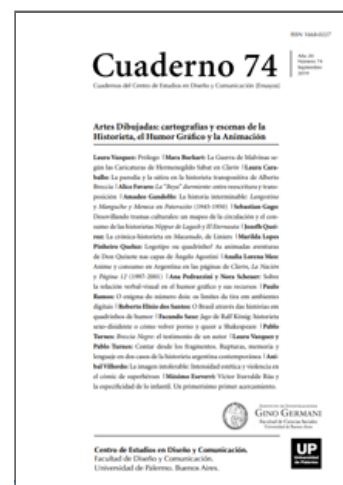

ISSN: 1668-0227

Artes Dibujadas:

cartografías y

escenas de la

Historieta, el Humor

Gráfico y la

Animación

Año XX, Septiembre 2019, Buenos Aires, Argentina | 256 páginas

descargar PDF

ver índice de la publicación

Ver todos los libros de la publicación

compartir en Facebook

Dra. Maria Graciela Rodríguez. Maestranda en Comunicación y Cultura, cohorte 2012-2013 (FCS-UBA). Licenciada en Ciencias de la Comunicación Social (2010). Facultad de Ciencias Sociales, Universidad de Buenos Aires (FCS-UBA). Coordinadora de la Red Iberoamericana de Investigadores en Anime y Manga (RIIAM).

La animación japonesa (アニメanime)1 es un arte audiovisual cuyo conocimiento en nuestro país fue escaso durante las décadas del '70 y '80 pero debido al incremento paulatino de su incorporación de las grillas de programación en canales infantiles y adolescentes en la televisión por cable -principalmente en Capital Federal y Gran Buenos Aires a comienzos de la década del ' 90 y principios del siglo XXI- su notoriedad fue creciendo en Argentina. Es por ello, que la prensa gráfica local comienza a plasmar en sus páginas información acerca de este producto cultural y su consumo.

A partir de lo planteado los objetivos son los siguientes: 
- Identificar y construir una muestra de notas periodísticas de los diarios Clarín, La Nación y Página 12 en el período 1997-2001 que tomen como eje central al anime (アニメ) y su consumo.

- Mencionar los soportes mediáticos en la cultura contemporánea a través de los cuales se produce la circulación del anime (アニメ) durante 1997-2001 en Argentina y su posterior consumo en la actualidad.

- Analizar las representaciones efectuadas en las notas periodísticas de la prensa gráfica (Clarín, La Nación y Página 12) sobre las representaciones del anime (アニメ) y su consumo local.

La selección de los periódicos se debe a: su alcance geográfico nacional, su amplio tiraje y a la presencia de artículos periodísticos en los cuales se trate como eje central a la animación japonesa y su consumo local. Además, consideramos que los tres diarios posean un repositorio digital que facilite la búsqueda del material para su posterior estudio. Cabe destacar que el análisis del artículo es cualitativo e interpretativo.

El período de análisis seleccionado coincide con la expansión de la televisión por cable en nuestro país, junto con la creación de canales argentinos y extranjeros dedicados a la transmisión de series de animación tanto de origen estadounidense como japonés. Asimismo, con la producción de fanzines, revistas, convenciones y ciclos de animación japonesa realizados tanto desde el fandom2 como desde editoriales argentinas y del ámbito académico. Destacamos que este artículo constituye un adelanto del capítulo cinco perteneciente a la tesis de maestría en Comunicación y Cultura (FCS-UBA): Anime mitológico y contemporáneo: pasajes, representaciones y construcciones de la(s) identidad(es) ‘Oriente-Occidente' (1995-2017). Producción, circulación y su consumo en Argentina. (Meo, en curso, FCSUBA) Dicha investigación tiene la intención de constituirse como una base para aquellos investigadores, docentes y fanáticos interesados en la indagación de la cultura japonesa en un producto cultural contemporáneo como el anime (アニメ), exponiendo tanto las condiciones de producción (en Japón), la circulación de este bien cultural, como el consumo de la animación nipona en nuestro país.

Con respecto al campo de las Narrativas Dibujadas coincidimos con Laura Vazquez (2009) al afirmar que trabajar tanto sobre animación como historieta y humor gráfico: “[...] nos sitúa en un campo epistemológico preciso. En este sentido, resulta necesario no leer sus objetos como 'pasados dorados' o 'nostalgias arcaicas' [sino que] [...] se trata de volver sobre las producciones para pensar su dimensión polémica y conflictiva" (p. 1).

Animación japonesa: producto de los medios masivos de comunicación

A continuación presentamos definiciones de anime (アニメ), los modos de circulación a través de los soportes mediáticos contemporáneos por los cuales fue y es consumido este arte dibujado y una breve mención a su llegada a nuestro país.

Mark MacWilliams plantea que el anime (アニメ) y la historieta nipona tienen una doble naturaleza híbrida. Por un lado, ambos combinan lo visual y lo verbal (Carrier, 2000, p. 69 en MacWilliams, 2008). Por otro, estos productos contemporáneos son híbridos culturales debido a la relación que Japón establece con Occidente. En ellos, se combinan la cultura visual Occidental con la tradición del arte secuencial y las caricaturas japonesas (Craig, 2007, p. 7; Nakamura 2003, p. 10 en MacWilliams, 2008). Otra forma de pensar al anime ( アニメ) es como un arquetipo cultural contemporáneo (Jung, 2002), pues, a través de él, podemos localizar vestigios del discurso mitológico antiguo adaptado a la cultura actual (Meo \& Goldenstein, 2011). 
Por su parte, Susan Napier (2005) considera que: "A diferencia de las caricaturas en Occidente, el anime en Japón es realmente un fenómeno mainstream pop cultural. [...] El anime es simplemente aceptado por virtualmente todas las generaciones de jóvenes japoneses como un artículo de primera necesidad cultural". (p. 7)3

Por lo tanto, con respecto a su público objetivo y su consumidor final, destacamos que la animación japonesa es producida tanto para niños y adolescentes como para adultos, ya que, debido a sus tramas tanto diversas como complejas junto a su calidad audiovisual, atrae a un público muy amplio (Meo y Goldenstein, 2011). William Tsutsui (2006) reflexiona que a partir de la década del '90 los productos de la cultura popular japonesa fueron cada vez más valorados por su gran calidad y por ser diferentes y refrescantes con respecto a las tradiciones narrativas y convenciones visuales de Hollywood.

Si bien en las últimas décadas el anime (アニメ) se ha difundido de forma notable hasta alcanzar su exportación masiva hacia Occidente, su llegada a países como Estados Unidos acontece en la década del '60 con la serie conocida como Astro boy (鉄腕 アトム Tetsuwan Atomu, 1963), sin embargo, tendremos que esperar un poco más de una década para su aparición en Argentina. (Meo, 2013)

Durante las décadas del ' 70 y ' 80 podemos destacar la transmisión por televisión abierta4 en nuestro país de animaciones del género shōnen (少年)5 : “Astroboy” (1976) (鉄腕アトム Tetsuwan Atom-Átomo, el brazo poderoso, 1963), “Fuerza G: la batalla entre planetas” (1979) (科学忍者隊ガッチャマンKagaku Ninjatai Gatchaman, 1972), “Grand Prix” (1980) (アロ ーエンブレム・グランプリの鷹 Arrow Emblem Grand Prix no takaArō Enbremu Guranpuri no taka, 1977), “Mazinger Z” (1981) (マジンガーZ Majingā Zetto, 1972-1974), etcétera. Otras destacadas producciones de la época pertenecientes al género shōjo (少女)6 son: “Heidi” (1977) (アルプス の少女八イジ Arupusu no Shōjo Haiji-Alps no Shōjo Heidi, 1974) y “La abeja Maya” (1983) (みつばちマーヤの冒 険 Mitsubachi Maya no boken, 1975-1976). La adquisición de estas animaciones niponas se debió a la compra de enlatados con el objetivo de disminuir costos y llenar horas de programación, ya que estos productos culturales fueron producidos para ser transmitidos y consumidos en el mercado nipón y al ser exportados a otros mercados (en el caso de Argentina con un desfase temporal importante entre su producción-transmisión japonesa y posterior transmisión y consumo en el exterior) los precios son mucho menores que en el mercado local.

Ross Mouer y Craig Norris (2009) retoman a los autores japoneses Kōichi Iwabuchi (1998) y Toshiya Ueno (1999) para hacer referencia a las estrategias de exportación de bienes culturales que las compañías audiovisuales niponas implementaban durante la década del '90. Indican que tanto Iwabuchi (1998) como Ueno (1999) plantean un proceso denominado 'la ausencia de la nacionalidad'. Este último, implica la eliminación de la presencia cultural japonesa en los personajes de anime (アニメ) y juegos de computadora. Este proceso lo relacionamos con el concepto de contrato de lectura (Verón, 1981), es decir, la relación entre el discurso de un determinado soporte y sus lectores. Se establece un nexo entre estas dos partes, un contrato. En el caso de la animación y la historieta japonesa, se plantea un discurso sin huellas de su procedencia social y cultural para apelar a diferentes lectores/consumidores y ello conlleva a un consumo global.

Generalmente cuando éramos niños/as y no tan pequeños/as, solíamos consumir varias horas de programación dedicadas a distintas animaciones desconociendo su procedencia, la cultura en torno a la cual fueron creadas y la época original de estreno. Nuestra fascinación giraba en torno por las diferentes tramas, los colores y diseños 
generales de los robots, la musicalización, por lo tanto, esos detalles no eran de importancia en aquel momento. Una de estas animaciones que captaba la atención del público no sólo de pequeños sino también adultos era: “Mazinger Z" (1981) (1972-1974, マジンガーZ Majingā Zetto), la historia tomaba como eje a un robot (Mazinger $Z$ ) y un grupo de científicos que lo utilizaban en su lucha en contra de las fuerzas maléficas del Dr. Hell (Doctor Infierno) quién quería gobernar el mundo.

En el año 1986 se emitía en la televisión abierta de Argentina, específicamente en canal 9 Libertad, "Robotech" (1985), una animación a la cual podemos referir como híbrida ya que se produce la combinación entre Japón como productor de las tres series que conforman "Robotech" (1985) y Estados Unidos como modelador de dichas animaciones cambiando ciertos aspectos de sus tramas para conformar una única. La combinación de estas tres animaciones japonesas: The Super Dimension Fortress Macross (超時空要塞マクロス Chō Jikū Yōsai Makurosu, 1982), Super Dimensional Cavalry Southern Cross (超時空騎団 サザンクロス Chōjikū Kidan Sazan Kurosu, 1984) y Genesis Climber Mospeada (機甲創世 記モスピーダ Kikō Sōseiki Mosupīda, 1983), que tenían en común robots transformables pero como anticipábamos no tenían conexión en sus tramas, genera no sólo rating sino la compra de merchandising (productos derivados de las series como por ejemplo juguetes) en torno a su éxito.

Consideramos que series como “Astroboy” (1976) (鉄腕アトム Tetsuwan Atom-Átomo, el brazo poderoso, 1963), “Mazinger Z” (1981) (1972-1974, マジンガーZ Majingā Zetto), “Heidi” (1977) (アルプスの少女ハイジ Arupusu no Shōjo Haiji-Alps no Shōjo Heidi, 1974), "Robotech" (1985), entre otras, abrieron una gran puerta en varias partes del mundo para una mayor exportación de anime (アニメ) en las décadas siguientes, combinando no sólo los bajos costos sino la calidad de los bienes culturales adquiridos.

La compra de enlatados durante las décadas del '70 y ' 80 se incrementa notablemente en los ' 90 . Alejandro Soifer (2012) plantea que esto se debió a la sanción de la Ley de Convertibilidad el 27 de marzo de 1991:

[...] y que duró hasta diciembre de 2001 con la caída del modelo económico que facilitó la entrada masiva de todo tipo de productos extranjeros en desmedro de la producción local, fue precisamente la década de la consolidación, el auge y la explosión de nuevos modos de consumo cultural que estuvieron íntimamente pegados a la imitación, la apropiación y la incorporación de los modelos estadounidense primero y luego japonés. (p. 111)

Sumado a la ventaja del 1 a 1, la masividad de este consumo cultural alcanza su apogeo en Argentina durante la década del '90 además con la expansión del cable -principalmente- en Capital Federal y Gran Buenos Aires. Varios canales como Cablín (1988-2000), Magic Kids (1995-2006), The Big Channel (1990-2001), entre otros, incorporaban a sus grillas de programación cada vez más horas de animación japonesa. (Meo, 2013)

En el caso de Cablín, algunas de las más destacadas animaciones japonesas transmitidas fueron: "Meteoro" ( ッ八GoGoGo, mahha gō gō gō, 1967), “Fuerza G: la batalla entre planetas” (科学忍者隊ガッチャマン Kagaku Ninjatai Gatchaman, 1972), Candy, Candy ( キャンディ・キャンディKyandi Kyandi, 1976), “Arbegas” o "Arbegas: el rayo custodio" (光 速電神アルベガス Kōsoku Denjin Arubegasu, 1983), "Los caballeros del zodíaco" (聖闘 士星矢 Seinto Seiya, 1986), Sailor moon (美少女戦士セーラームーンBishōjo Senshi Sērā Mūn, 1992), entre otras. 
El incremento de la popularidad del anime (アニメ) en nuestro país no sólo se debe a su aparición en las programaciones de la televisión abierta y cable facilitados por la convertibilidad, sino a diversos factores entre los que podemos destacar: el surgimiento de editoriales productoras de revistas dedicadas a la difusión del anime (アニメ) y el manga (Lazer, producida y editada por Ivrea es uno de los casos más destacables), ciclos de proyección de anime (アニメ), eventos de cosplay7 en Capital Federal por ejemplo, la concurrencia a festivales vinculados con la comunidad nipona realizados en el Jardín japonés, editoriales dedicadas a la comercialización de manga como Ivrea, dirigida por Leandro Oberto ex conductor del "El club del animé"8 -programa de cable emitido por Magic kids-, etcétera. (Meo, 2016)

Asimismo, consideramos que debido a la globalización y a la creación de las Nuevas Tecnologías de Información y Comunicación (NTIC) a comienzos del siglo XXI los soportes mediáticos se incrementan y los tiempos entre la producción, circulación y consumo de estos productos culturales japoneses se han acortado notablemente, como así también han cambiado las formas de consumirlos con productos tecnológicos como notebooks, PC de escritorios y/o celulares conectados a Internet se puede acceder a diversas páginas web dedicadas a la difusión del anime (アニメ) y consumirlo de forma gratuita en la mayoría de los casos (Meo, 2015). Con respecto a los celulares, a través del sistema operativo Android, por ejemplo, se pueden descargar desde la Play store diversas aplicaciones para consumir anime (アニメ), manga, música de anime, radios vinculadas a este tipo de productos culturales: AnimeEsp, JPAnime!, Música Anime, Anime Radio, Manga rock, ZingBox (Z manga), etc.

Por lo tanto, la circulación de estos productos se ha expandido notablemente y tanto el anime (アニメ) como el manga (漫画) han tomado gran notoriedad no sólo en nuestro país sino alrededor del globo, como afirma Napier (2011):

Hace veinte años, cualquier no-japonés encontrando las palabras "anime" o "manga" habría sacudido su cabeza con perplejidad. Mirando hacia adelante en el siglo veintiuno, nos encontramos en un mundo donde el "anime" y el "manga" son omnipresentes, conocidos y amados alrededor del mundo desde Sudáfrica a Latinoamérica. ( $p$. 226) 9

Representaciones de la prensa gráfica

En este apartado retomamos artículos destacando ciertas informaciones que den cuenta de las representaciones y el conocimiento que se tenía durante el período 1997-2001 acerca del anime (アニメ) como así también ciertos datos que generen en los lectores desinformación por erratas, por ejemplo.

Con respecto al término representación nos referimos al mismo así como lo plantea Carlo Ginzburg (2000), es decir, por sustitución del objeto/persona ausente o por evocación mimética:

[...] los maniquíes de cera, de madera o de cuero que se colocaban sobre el catafalco real durante los funerales de los soberanos franceses o ingleses con el lecho fúnebre vacío y cubierto por un lienzo mortuorio que aún más antiguamente 'representaba' al difunto soberano. La voluntad mimética presente en el primer caso estaba ausente en el segundo, pero en ambos se hablaba de 'representaciones. (p. 85)

Asimismo, retomamos a Fred Patten (2004) pues realizó una recopilación de 25 años de sus ensayos y críticas para plasmarlos en su libro Watching anime, Reading manga. 25 Years of Essays and Reviews (Mirando anime, 
y Leyendo manga. 25 años de Ensayos y Críticas), estas notas estaban vinculadas con estos productos culturales nipones. Coincidimos con su pensamiento de dejar los ensayos en su libro tal cual fueron originalmente escritos, aun conservando los errores, pues de esta manera se puede vislumbrar el estado de conocimiento acerca del anime (アニメ) en Estados Unidos en la época que fueron escritos. Como planteábamos hace unas líneas, lo mismo sucede con la muestra de notas periodísticas seleccionadas para el análisis, en las mismas se hacen presentes aciertos y desaciertos en la información. Comenzamos con un artículo escrito por Fabiana Scherer (1997) para La Nación, en el mismo consideraba que hay un incremento de señales infantiles que se incorporaban a la televisión por cable argentina. En este caso destacamos el anuncio en julio de 1997 del arribo a la pantalla chica de Locomotion (1996-2005). Canal que si bien Scherer (1997) considera destinado para la familia, era una señal focalizada en la transmisión de animaciones para jóvenes adultos y en determinados horarios para adultos. Aunque cabe manifestar que sí hace hincapié en la organización por segmentos de las animaciones: "[...] Locomotion organiza los dibujos animados en segmentos bien diferenciados y complementarios entre sí" (p. 1). Otro desacierto ocurre cuando la autora plantea que la animación japonesa en esa época va ganando espacios tanto en la televisión abierta como en cable pero al mencionar como ejemplo a Dragon Ball (ドラゴンボールDoragon Bōru - Las esferas del dragón, 1986-1989), afirma que su procedencia es de medio oriente y no lejano oriente, quedando en evidencia el desconocimiento de su origen.

Máximo Eseverri (1998) desde la redacción de Página 12 comunicó a los fanáticos de la animación japonesa sobre un “ciclo de animé" en el cual proyectaron Macross II - 超時 空要塞マクロスII Lovers again-Chôjikû Yôsai Macross II Lovers, Again - Super dimensional Fortress Macross II: Lovers again - La Fortaleza Super dimensional Macross II: Amantes de nuevo, compuesta por seis OVA (Original Video Animation - Animación Original en Video) -en el auditorio del Centro Cultural Rojas. También recuerda que en 1997, el Centro se convierte en el primer lugar de Capital Federal en donde los fans pueden ver estás películas en pantalla grande y con buen sonido, pues, durante la primavera se proyectaron según sus palabras: “[...] joyas del género [...] obras imprescindibles [...]" (p. 1) de Katsuhiro Ōtomo10, Mamoru Oshii11 y Hayao Miyazaki12. Y por último, afirma que en las últimas funciones de 1997 se colmaba la capacidad de la sala, dando cuenta del creciente fanatismo en esa época y la posibilidad de contar con un espacio que proyecte estos bienes culturales. Además, menciona a la extinta comiquería Camelot que facilitó las películas en ese momento. Esta última funcionó durante muchos años y se había convertido en el lugar de ensueños para aquellos que buscaban este tipo de materiales.

Con el avance de la tecnología en general y principalmente con Internet se pueden consumir animaciones niponas de diversidad de géneros, directores, tramas narrativas etcétera, pero generalmente los productos que llegan a la pantalla de cine y principalmente durante el período que hemos elegido para el análisis, son productos mainstream y que no necesariamente tienen la calidad de la obras de los ya mencionados Ōtomo, Oshii y Miyazaki. Esto es algo que destaca Diego Lerer (1999) en su crítica a la película Dragon Ball Z: El poder Invencible-Dragon Ball Z II: The Burning Battles (1993) ya que la considera monótona, con violentos combates, aburrida y con limitados recursos visuales al compararlas con obras como La princesa Mononoke (もののけ姫 Mononoke Hime, 1997) o Akira (アキラ Akira, 1988), por ejemplo. Una errata es el año de estreno en Japón indicada en el artículo como 1990 cuando en realidad fue en 1993.

Con respecto al modelo industrial y creativo de la animación, generalmente se pone en comparación el modelo propio con el constituido por Disney por ser uno de los estudios más antiguos y exitosos en estos menesteres, 
en el caso de la animación argentina Cóndor Crux (2000), uno de sus creadores Carlos Ruiz Brussain entrevistado por Lorena García (1999) para La Nación, manifiesta que: “Un exceso de movimientos encarece mucho una película de animación", es por ello que su equipo tomó de guía el proceso efectuado en la animación japonesa que surge por la limitación de presupuesto y la limitación de movimientos en escena. El director consideró que ese estilo también es imitado por Estado Unidos dando prioridad a la expresividad de los personajes y no haciendo hincapié en la desmesura de los movimientos. Por lo tanto, estos cambios tomados de la animación nipona los vincula con la tradición cultural y estética japonesa.

Mariana Enriquez (2000) planteaba en Página 12 que: “[...] los canales de aire ya tienen programas especializados en anime [...] "Animérica”, por América [...] ciclos de anime japonés en el Centro Cultural Ricardo Rojas [...] y Locomotion, el canal de DirecTV especializado en animación para adultos [...]" (p. 1). Dando un panorama alentador del progreso del anime (アニメ) con respecto a su circulación y potencial consumo en nuestro país, aunque en esta época el consumo aun no se había consolidado. Cabe realizar una aclaración cuando Enriquez (2000) se refiere a "ciclos de anime japonés", en Japón el término anime (アニメ) hace referencia a la animación de todo tipo de procedencia, en cambio en Occidente cuando hablamos de anime ( $\mathcal{P}$ 二メ) hacemos referencia a la animación del país nipón, por lo tanto, no hacía falta aclarar su origen.

Además de los soportes mediáticos de la época, también se hace referencia a los espacios de socialización de los fanáticos. Enriquez (2000) planteaba que en Fantabaires -convención de cómics realizada en Buenos Aires, cuya primera edición fue en 1996- se producía ese año una aparición masiva del Hentai (変態 género pornográfico de manga y アニメ anime) y que:

El reciente furor por la animación japonesa es notable cuando se ve el crecimiento de Editorial Ivrea, editora de la revista de animación japonesa Lazer y la primera en editar fielmente y con continuidad los mangas de Ranma $1 / 2$, Evangelion, [...] y Fushigi Yuugi. (p. 1)

Laura Gentil (2001) en su artículo para Clarín, también da cuenta de un evento muy importante que convoca a los fanáticos de los cómics, historietas argentinas, anime (アニメ) y manga, llamado ExpoComics \& Animé, una feria anual realizada en la rural del 23 de noviembre al 2 de diciembre de 2001.

Gentil (2001) manifiesta que:

[...] En la exposición se podrán ver más de 100 originales de manga y animé. Para neófitos, así se llaman las historietas y dibujos animados japoneses de los años 90 , protagonizadas por personajes con superpoderes que se mueven en un mundo de violencia y corrupción. (p. 1)

En esta cita podemos observar nuevamente la calificación y el estereotipo de clasificar a los productos nipones como contenedores de violencia, como hemos manifestado hasta el momento, las trama dependerán del género de la animación o la historieta, obviamente no todas están inclinadas hacia la violencia, la corrupción o la monotonía. Otra cuestión a remarcar es la indicación temporal que la autora le marca al lector: "[...] las historietas y dibujos animados japoneses de los años 90 [...]", consideramos que efectúa esta aclaración debido al incremento de estos bienes culturales en nuestro país durante esa década, como indicamos con anterioridad, aunque el origen del anime (アニメ) se remonta a comienzos del siglo XX. 
A modo de cierre, a través de las distintas notas periodísticas hemos podido reconstruir una primera mirada hacia el incremento de las opciones para ver anime (アニメ) en televisión abierta y cable durante el período 1997-2001; las diferentes proyecciones en el Centro Cultural Rojas, haciendo hincapié en animaciones japonesas de elite, es decir, realizando una selección minuciosa de aquellas "joyas" a exhibir en sus salas o las comparaciones de obras maestras de anime (アニメ) ya consolidas con productos muy populares pero de menor calidad audiovisual y de casi una década de antigüedad, ya que su proyección en Argentina fue posterior a su estreno en Japón.

Asimismo, se observaron las representaciones del anime (アニメ) como inferior en calidad técnica con respecto a productos de otras procedencias, también se remarcó lo positivo que constituye su estilo de animación porque disminuye los costos y se observó el error de asumir que las tramas en general son violentas. A su vez, destacamos que se presentan en las notas, tanto el incremento de los espacios de difusión de estos bienes culturales que posibilitaban un mayor consumo y aumento de posibles fanáticos; la incorporación de la animación e historieta nipona en áreas pertenecientes al cómic e historietas nacionales y por último, el crecimiento de uno de los pilares de este consumo en Argentina, editorial Ivrea. En contraposición con los múltiples modos de acceso para consumir en la actualidad, en décadas pasadas, estábamos limitados al alquiler y/o compra de VHS, proyecciones muy esporádicas, o a la proyección y transmisión de los anime (アニメ) mainstream tanto en el cine como en la televisión. La convertibilidad y la aceptación del público propiciaron una oleada aún mayor de la animación japonesa, aunque el desconocimiento de su procedencia o la diversidad de formatos de consumo y géneros eran aún una constante en nuestro país.

Por lo tanto, en estas líneas pudimos vislumbrar, como planteaba Patten (2004), el estado de conocimiento acerca de este producto cultural nipón, sólo que en esta oportunidad ese viaje al pasado fue a través de una segmento de la prensa gráfica argentina.

\section{Notas}

1. Todos los términos japoneses utilizados en este artículo, como por ejemplo anime (アニメ), es escrito sin la s en su forma plural. Las palabras extranjeras en el cuerpo del texto han sido escritas en cursiva y traducidas. Asimismo, se ha incorporado en el caso de las palabras japonesas, su correspondiente kanji (漢字) y en algunas oportunidades el hiragana dependiendo de su utilización vox populi. Los kanji (漢字 carácter chino) son sinogramas empleados en la escritura del idioma japonés, es uno de los tres sistemas de escritura en Japón, los otros dos son silabarios: hiragana (平仮名 oひらがな) y katakana (片仮名 ○ カタカナ). Este último suele ser utilizado para la escritura de palabras tomadas de otros idiomas, como ejemplo el inglés. La palabra anime (ア二 メ) proviene del término inglés animation (アニメーション, animēshon-animación) y de ahí su abreviatura anime (アニメ). Por lo tanto, su escritura es en katakana (片仮名 ○ カタカナ). En algunas oportunidades se ha incluido rōmaji (caracteres romanos), es decir, la escritura de la lengua japonesa en letras romanas o latinas. Además, las vocales largas se indican con un macrón encima de la vocal (̄̄).

2. Conjunto de aficionados que comparten un pasatiempo o gusto por una serie, película, personaje en particular, etcétera.

3. La traducción es propia. En el original en inglés: "Unlike cartoons in the West, anime in Japan is truly a mainstream pop cultural phenomenon. [...] anime is simply accepted by virtually all the younger generation of 
Japanese as a cultural staple".

4. Los datos referentes a las fechas de transmisión de las animaciones fueron extraídos de los libros de Jorge Nielsen (2006-2010): "La magia de la televisión argentina" del número 3 al 7. Con respecto a estas fechas hemos indicado en el primer paréntesis el año en que fue estrenado en Argentina y en el segundo paréntesis junto a su nombre en japonés figura la fecha en que fue transmitido por primera vez en Japón. Además, los anime (アニメ) son citados primero cómo son conocidos y mencionados en los listados de programación en Argentina.

5. Generalmente sus tramas tienen mucha acción y humor. Sus protagonistas suelen ser masculinos y la audiencia objetivo serían varones pre-adolescentes y adolescentes (menores de 18).

6. Su trama es romántica y se focaliza en los sentimientos de los protagonistas. La audiencia objetivo serían mujeres pre-adolescentes y adolescentes (menores de 18).

7. El costume play (“Juego de disfraz”- cosplay es la forma abreviada y como se utiliza el término comúnmente) consiste en disfrazarse como un personaje de manga, anime (アニメ) o personajes de películas, cómics, etcétera.

8. Comienza a transmitirse en 1998. Fue producido por PRAMER y una productora argentina llamada La Corte. Su primer conductor fue Leandro Oberto también fundador de la revista Lazer (1997-2009) que contaba con publicaciones orientadas al manga (漫画) y al anime (アニメ). Luego fue reemplazado por Mariela Carril egresada de la licenciatura en Ciencias de la Comunicación, en la Facultad de Ciencias Sociales (UBA), también formó parte del staff en la revista dirigida por Oberto- debido a comentarios que hizo sobre el canal. Dicho programa era transmitido los días sábados y domingos a la noche en el cual pasaban series antiguas y relativamente contemporáneas, además de algunas notas de interés para los otaku (オタク), es decir, fanáticos del manga 漫画 y el anime アニメ. Algunos sujetos no sólo consumen estos productos o juegan videojuegos basados en estos bienes culturales sino que realizan prácticas que podríamos llamar "activas" al efectuar, por ejemplo, el subtitulado no oficial de anime (アニメ) y manga, es decir, el fansub (fan subtittled-subtitulado de fanáticos llamados fansubbers) o vestirse y actuar como lo hacen determinados personajes (cosplayers). Hacer una determina actividad, no inválida la realización o combinación de otras.

9. La traducción es propia. En el original en inglés: "Twenty years ago, any non-Japanese encountering the words "anime" or "manga" would have shaken their heads in puzzlement. Flash forward to the twenty-first century, and we find a world where anime and manga are ubiquitous, known and loved around the globe from South Africa to Latin America".

10. Guionista, director de anime (アニメ) y dibujante de manga.

11.Guionista, director de anime (アニメ), novelista, escritor y dibujante de manga.

12. Director, productor, guionista, de anime (アニメ) y dibujante de manga.

Bibliografía 
Ginzburg, C. (2000). "Representación. La Palabra, la idea, la cosa”. En Ojazos de Madera. Barcelona: Península.

MacWilliams, M. W. (2008). Introduction. En Japanese Visual Culture: Explorations in the World of Manga and Anime. Armonk, NY: M. R. Sharpe.

Meo, A. L. y Goldenstein, B. A. (2011). “Introducción” en Construcción del mito en la animación japonesa. Su relación con la tecnología, los mass media y la naturaleza (Tesis de licenciatura). Facultad de Ciencias Sociales, Universidad de Buenos Aires. Aprobada en Junio de 2010. Recuperada de http://comunicacion.sociales.uba.ar/? page_id=636 N² 2356. (Consultado: 25/02/16).

Meo, A. L. (2013). Autoconciencia de la vorágine alienante y racionalizadora de la modernidad. Análisis del cortometraje 'Carne de cañón'. Jornadas de la Carrera de Ciencias de la Comunicación "Comunicación y Ciencias Sociales. Legados, diálogos, tensiones y desafíos”. GT 4 Narrativas dibujadas: historieta, humor gráfico y animación. Facultad de Ciencias Sociales. Universidad de Buenos Aires. 27, 28 y 29 de noviembre de 2013. Recuperado de https://www.academia.edu/7960044/Meo_Analia_Lorena_2013_Auto conciencia_de_la_vor\%C3\%A1gine_alienante_y_racionalizadora_de_la_modernidad. _An\%C3\%A1lisis_del_cortometraje_Carne_de_ca\%C3\%B1\%C3\%B3n_._Jornadas_ de_la_Carrera_de_Ciencias_de_la_Comunicaci\%C3\%B3n._FCS-UBA_(Consultado: 1/03/16).

Meo, A. L. (2015). “Animación japonesa. Industrias culturales, medios masivos de comunicación y productos de la cultura pop nipona”. Questión, Vol. 1, N 45, 358-372. Recuperado de http://perio.unlp.edu.ar/ojs/index.php/question/article/view/2389/2118 (Consultado: 28/02/16).

Meo, A. L. (en curso). Anime mitológico y contemporáneo: pasajes, representaciones y construcciones de la(s) identidad(es) ‘Oriente-Occidente' (1995-2017). Producción, circulación y su consumo en Argentina. (Tesis de maestría). Facultad de Ciencias Sociales, Universidad de Buenos Aires.

Meo, A. L. (2016). "Aproximaciones al anime: producción, circulación y consumo en el siglo XXI”. Questión, Vol. 1, N ${ }^{\circ}$ 51, 251-265. Universidad Nacional de La Plata, La Plata, Argentina. Recuperado de http://perio.unlp.edu.ar/ojs/index.php/question/article/ view/3416/2874

Mouer, R. y Norris, C. (2009). Exporting Japan's culture: From management style to manga. En Sugimoto, Y. (Ed.), The Cambridge Companion to Modern Japanese Culture. E.E.U.U.: Cambridge University Press.

Napier, S. J. (2005). “Why anime?” en Anime from Akira to Howl's Moving Castle. Experiencing Contemporary Japanese Animation. New York: Palgrave Macmillan.

Napier, S. J. (2011). “Manga and anime. Entertainment, big business, and art in Japan”, en Routledge Handbook of Japanese Culture and Society. Editado por Victoria Lyon Bestor y Theodore C. Bestor, con Akiko Yamagata. New York: Routledge. Nielsen, J. (2006). La magia de la televisión argentina 3: 1971-1980: cierta historia documentada. Buenos Aires: Del Jilguero. Nielsen, J. (2007). La magia de la televisión argentina 4: 1981-1985: cierta historia documentada. Buenos Aires: Del Jilguero. Nielsen, J. (2008). La magia de la televisión argentina 5: 1986-1990: cierta historia documentada. Buenos Aires: Del Jilguero. Nielsen, J. (2009). La magia de la televisión 
argentina 6: 1991-1995: cierta historia documentada. Buenos Aires: Del Jilguero. Nielsen, J. (2010). La magia de la televisión argentina 7: 1996-2000: cierta historia documentada. Buenos Aires: Del Jilguero.

Patten, F. (2004). "Preface" en Watching anime, Reading manga. 25 Years of Essays and Reviews. Berkeley: Stone Bridge Press.

Soifer, A. (2012). "La felicidad del 1 a 1" en Que la fuerza te acompañe. La invasión de las culturas nerd, geek y friki. Buenos Aires: Marea Editorial.

Tsutsui, W. (2006). “Introduction”. En Tsutsui, W. y Ito, M. (Comps.). Godzilla's footsteps. Japanese pop culture. Icons on the global stage. E.E.U.U.: Palgrave Macmillan.

Vazquez, L. "Editorial”. Narrativas dibujadas: arte, cultura de masas y lenguaje. Coordinadora de número de Diálogos de la Comunicación. (Coordinación de los 18 artículos de la edición, presentación editorial en formato video y en texto, producción de entrevistas a referentes del campo y fotorreportaje). Revista académica de la Federación Latinoamericana de Facultad de Comunicación Social, FELAFACS, Lima, julio de 2009. Con Comité de Referato Internacional y Arbitraje (ISSN 1995- 6630). Recuperado de http:// www.dialogosfelafacs.net/wpcontent/uploads/2011/10/78.pdf (Consultado: 15/02/16).

Verón, E. (1981). La Semiosis Social. Barcelona: Gedisa.

\section{Animaciones mencionadas}

Bossi, P. y Buscarini, J. P. (Productores). y Buscarini, J. P., Glecer, S. y Holcer, P. (Directores). (2000). Cóndor Crux [Película]. Argentina: Patagonik Film Group.

Hasegawa, Y. (Director). (1984). Super Dimensional Cavalry Southern Cross (超時空騎団 サザンクロス Chōjikū Kidan Sazan Kurosu). [Serie de televisión]. Japón.

Hayashi, S. (Director). (1977-1978). Grand Prix (アローエンブレム・グランプリの鷹 Arrow Emblem Grand Prix no taka-Arō Enbremu Guranpuri no taka). [Serie de televisión]. Japón: Fuji TV.

Imada, CH. y Anzai, Y (Productores). y Hashimoto, M (Director). (1993). Dragon Ball Z: El Poder Invencible (ドラ ゴンボールZ 燃えつきろ!!熱戦・烈戦・超激戦Doragon Boru Zetto: Moetsukiro!! Nessen - Ressen - Chōgekisen) [Película]. Japón: Toei Company.

Ishiguro, N. (Director). (1982-1983). The Super Dimension Fortress Macross (超時空要塞 マクロスChō Jikū Yōsai Makurosu). [Serie de televisión]. Japón: Mainichi Broadcasting.

Kuri, I. y Barron, R. V. (Directores). (1985). Robotech. [Serie de televisión]. Estados Unidos y Japón.

Morishita, K. (Director). (1983-1984). Arbegas o Arbegas: el rayo custodio (光速電神アル ベガス Kōsoku Denjin Arubegasu). [Serie de televisión]. Japón: TV Tokyo. 
Morishita, K. y Kikuchi, K (Directores). (1986-1989). Los caballeros del zodíaco (聖闘士星 矢 Seinto Seiya, 1986) [Serie de televisión]. Japón: TV Asahi.

Nishiyama, A. (Director). (1972-1974). Fuerza G: la batalla entre planetas (科学忍者隊ガ ッチャマン Kagaku Ninjatai Gatchaman). [Serie de televisión]. Japón: Family Gekijō y Fuji TV.

Okazaki, M. y Nishio D. (Directores). (1986-1989). Dragon Ball (ドラゴンボール Doragon Bōru - Las esferas del dragón). [Serie de televisión]. Japón: Fuji TV.

Saitō, H., Kaminashi, M y Endō, S. (Directores). (1975-1976). La abeja Maya (みつばちマーヤの冒険 Mitsubachi Maya no boken). [Serie de televisión]. Japón: TV Asahi.

Sasagawa, H. (Director). (1967-1968). Meteoro (マッ八GoGoGo, mahha gō gō gō). [Serie de televisión]. Japón: Fuji TV.

Sato, J., Ikuhara, K. y Igarashi, T. (Directores). (1992-1997). Sailor moon (美少女戦士セーラームーンBishōjo Senshi Sērā Mūn). [Serie de televisión]. Japón: TV Asahi.

Serikawa, Y., Katsuda, T. y Katsumata, T. (Directores). (1972-1974). Mazinger Z (マジンガ —Z Majingā Zetto). [Serie de televisión]. Japón: Fuji TV.

Suzuki, R. y Katō, S. (Productores). y Ōtomo, K. (Director). (1988). Akira (アキラ Akira) [Película]. Japón: TMS Entertaiment, Bandai, Kōndasha, Mainichi Broadcasting System, Sumitomo Corporation y Tohō.

Suzuki, T. (Productor). y Miyazaki, H. (Director). (1997). La princesa Mononoke (ものの け姫 Mononoke Hime) [Película]. Japón: Studio Ghibli.

Takahata, I. (Director). (1974). Heidi (アルプスの少女ハイジ Arupusu no Shōjo Haiji-Alps no Shōjo Heidi). [Serie de televisión]. Japón: Fuji TV.

Tezuka, O. (Director). (1963-1966). Astroboy (鉄腕アトム Tetsuwan Atom-Átomo, el brazo poderoso). [Serie de televisión]. Japón: Fuji TV.

Yamada, K. (Director). (1983-1984). Genesis Climber Mospeada (機甲創世記モスピーダ Kikō Sōseiki Mosupīda). [Serie de televisión]. Japón: Fuji TV.

Yatagai, K. (Director). (1992). Super dimensional Fortress Macross II: Lovers again (超時空 要塞マクロスII Lovers again-Chôjikû Yôsai Macross II Lovers, Again - La Fortaleza Super dimensional Macross II: Amantes de nuevo). [OVA]. Japón: AIC y Oniro.

Yukimuro, SH. (Director). (1976-1979). Candy, Candy (キャンディ・キャンディ Kyandi Kyandi). [Serie de televisión]. Japón: TV Asahi. 
Sadamoto, Y. (1994-2013). Neon Genesis Evangelion (新世紀エヴァンゲリオン Shin Seiki Evangerion). Volúmenes (1-14). Japón: Kadokawa Shoten.

Takahashi, R. (1987-1996). Ranma 1⁄2 (らんま1⁄2 Ranma Nibun no Ichi). Volúmenes (1-38). Japón: Shōgakukan. Watase, Y. (1992-1996). Fushigi Yūgi (ふしぎ遊戯 El juego misterioso). Japón: Shōgakukan.

Notas periodísticas

Enriquez, M. (11 de diciembre de 2000). Arrancó Fantabaires 2000, una cita de tribus urbanas. Los fantasmas están entre nosotros. Página 12. Recuperado de http://www. pagina12.com.ar/2000/00-12/00-12-11/pag25.htm

Enriquez, M. (28 de octubre de 2000). Las dos caras del Japón, en un anime que juega al apocalipsis. Página 12. Recuperado de http://www.pagina12.com.ar/2000/00-10/00-10- 28/pag24.htm

Eseverri, M. (14 de mayo de 1998). ¡Buenas noticias para el amigo comiquero! .Página 12. Recuperado de http://www.pagina12.com.ar/1998/suple/no/98-05/98-05-14/199.htm

García, L. (27 de marzo de 1999). La Argentina dibujada. La Nación. Recuperado de http:// www.lanacion.com.ar/132749-la-argentina-dibujada

Gentil, L. (22 de noviembre de 2001). Lanzan una gran muestra de comics e historietas de culto. Clarín. Recuperado de http://edant.clarin.com/diario/2001/11/22/s-03901.htm

Lerer, D. (7 de enero de 1999). Superhéroes a la japonesa. Clarín. Recuperado de http:// edant.clarin.com/diario/1999/01/07/c-00401d.htm

Scherer, F. (7 de agosto de 1997). La TV: cosa de chicos. La Nación. Recuperado de http:// www.lanacion.com.ar/74396-la-tv-cosa-de-chicos

Abstract: This article approaches a first approximation about the representations made by the Argentinean newspapers Clarín, La Nación and Página 12 referring to the Japanese animation (アニメ anime) and its consumption in Argentina during the period 1997-2001.

Key words: anime - Argentina - Argentinean newspapers - representations - consumption.

Resumo: Este artigo aborda uma primeira aproximação sobre as representações feitas pelos jornais argentinos Clarín, La Nación e Página 12, referindo-se à animação japonesa ( アニメanime) e ao seu consumo na Argentina durante o período 1997-2001. e no início do século XXI.

Palavras chave: anime - Argentina - jornais argentinos - representações - consumo.

[Las traducciones de los abstracts al inglés y portugués fueron supervisadas por el autor de cada artículo] 
Anime y consumo en Argentina en las páginas de Clarín, La Nación y Página 12 (1997-2001) fue publicado de la página 109 a página122 en Cuadernos del Centro de Estudios de Diseño y Comunicación №74 